\title{
Linguistische Spezifizierung des Untersuchungsgegenstandes
}

Da ich für die vorliegende Arbeit eine interdisziplinäre Betrachtung der Daten aufgrund ihres Entstehungskontextes als zentral erachte und aus diesem Grund neben den linguistischen Beschreibungsebenen insbesondere wirtschaftswissenschaftliche Hintergründe einbringe - und literaturwissenschaftliche Konzepte einbeziehe -, stelle ich den Analyseergebnissen ein Kapitel zur linguistischen Verortung des Untersuchungsgegenstandes voran. Dieses Kapitel umfasst maßgebende linguistische Merkmale der erhobenen Daten sowie die Zuordnung der Daten in den textlinguistischen Fachbereich. In dem daran anschließenden dritten Kapitel werden in Folge erzähltheoretische Ansätze in den Blick genommen. Das Ziel des nun folgenden Kapitels ist es, den Untersuchungsgegenstand auf der Basis maßgebender gemeinsamer Merkmale zu erfassen. Da die Erzählungen nicht ausschließlich auf Schrifttexten beruhen, gehe ich im ersten Unterkapitel darauf ein, wie sie dennoch im Rahmen der Textlinguistik verortet werden können (Abschn. 3.1). So ist doch die Textlinguistik prinzipiell der linguistische Fachbereich, dem die disziplinäre Zuständigkeit für die Analyse von Narrativität unterliegt (Lehmann 2012: 170). Im Anschluss daran folgt eine Beschreibung gemeinsamer Charakteristika des gesamten Vermittlungsvorkommens auf zwei Ebenen. Auf der ersten Ebene beziehe ich mich auf textlinguistische Merkmalsbereiche, um die Daten im linguistischen Diskurs konkret zu verorten (Abschn. 3.2). Auf der zweiten Ebene behandle ich pragmatische Spezifika aus der linguistischen Perspektive, die daraus resultiert, dass die erhobenen (S)D in direkter Nähe zur Werbung für das unternehmenseigene und im Prozess der Corporate Identity erhobene „Unternehmens-Selbst“ stehen. Daher handelt das dritte Unterkapitel davon, inwiefern die Daten Gemeinsamkeiten und Unterschiede zur Werbung aufweisen. 


\subsection{Verortung der Daten unter der textlinguistischen Perspektive}

Wie die multimodale Beschaffenheit der Daten im textlinguistischen Diskurs eingeordnet werden kann, lässt sich mit einem kurzen Schlaglicht auf die Entwicklung der Textlinguistik im Zuge der sich verändernden „Textwelten“ verdeutlichen. Damit ich der Zielsetzung des Kapitels, die Daten näher zu spezifizieren, nachkomme, wende ich diese Erkenntnisse und Forderungen immer wieder auf das erhobene Korpus an.

Die Sprachwissenschaft betrachtet Erzählen als ein sprachlich komplexes Verfahrensmuster meist in Abgrenzung zur Deskription und Argumentation, welches die Rezipient/innen aufgrund eines spezifischen ,globalen Musters“ (Heinemann/ Viehweger 1991: 237) oder einer bestimmten thematischen Entfaltung (Brinker et al. 2014: 60 f.) erkennen. War die frühe linguistische Erzählforschung von der Literaturwissenschaft und vom Strukturalismus geprägt und auf literarische, also vorwiegend schriftliche Erzählungen ausgerichtet (vgl. Gülich/Hausendorf 2000: 370, Adamzik 2016: 19), änderte sich diese Perspektive im Zuge der pragmatischen Wende, die ihre Hauptwirkung in der Sprachwissenschaft in den 1970er Jahren entfaltete (Feilke 2000: 64). Sie zeichnet sich durch den Wechsel „,von der systemorientierten zur kommunikations- und funktionsbezogenen Sprachbetrachtung aus" (Fix 2008a: 15, vgl. auch Diatlova 2003: 11 ff.). Für die Frage, was nun als Text gilt, ist diese Entwicklung von Bedeutung, denn innerhalb dieser Entwicklung geriet zusätzlich die Sprachverwendung - insbesondere mündlicher Realisierungen - in den Blick der Linguistik. Die Debatte über die Definition von Texten hat das insofern befeuert, als das nach kurzer Zeit die grundlegende Frage zur Diskussion stand, ob mündliche Realisierungen Texte sind und von daher überhaupt in den Zuständigkeitsbereich der Textlinguistik fallen. Wurde noch in der Anfangsphase der (deutschen) Textlinguistik das Mündliche ebenfalls als Text aufgefasst, wie die zwei folgenden Zitate verdeutlichen:

„Es wird, wenn überhaupt gesprochen wird, nur in Texten gesprochen.“ (Hartmann 1971: 12)

„Ein Text ist ein Stück mündlicher und schriftlicher Rede [...].“ (Schmidt 1977: 155)

hat sich das heute insofern verändert, als mündliche Realisierungen im deutschsprachigen Raum (anders z. B. in der englischsprachigen Tradition) weniger 
Untersuchungsobjekte der Textlinguistik sind (Adamzik 2016: 70, 86). Die unterschiedliche Gewichtung der Perspektiven und Beschreibungsansätze führte zu verschiedenen Forschungsrichtungen mit ihren eigenen Untersuchungsmethoden, die je nachdem entweder die Interaktion, den Erzähltext oder die Geschichte fokussieren (Gülich/Hausendorf 2000: 370 ff.). Da beim mündlichen Erzählen die Interaktion im Mittelpunkt des Interesses steht, ist es für gewöhnlich Untersuchungsgegenstand der (ethnomethodologischen) Konversationsanalyse, der Ethnographie des Sprechens und ,verschiedener Spielarten der linguistischen Diskursanalyse“ (Quasthoff 2001: 1296). Während hingegen die Textlinguistik das Erzählen bezogen auf den Erzähltext und die Geschichte untersucht. In beiden Fällen handelt es sich jedoch um zwei Realisierungsformen oder - nach Stöckls „Netzwerk der Modalitäten“ (2004: 18) - mediale Varianten von Sprache und damit auch von Texten. Diese Sichtweise teilen nicht alle Linguist/innen. Ehlich (1983b) argumentiert gegen gesprochensprachliche Texte, da mit dem Terminus ,Text“ die „Konservierung einer Äußerungsstruktur“ (Schwitalla 2006: 192) eng verbunden sei, die bei komplex mündlichen Interaktionsformen nicht gegeben ist. Eine „natürliche“ mündliche Interaktion ist situativ gebunden und kann entgegen eines Textes - nicht außerhalb des Entstehungsortes rezipiert werden. Zusätzlich fügt Rolf (1993) hinzu, dass an dialogischen Äußerungsketten mehrere Sprecher/innen beteiligt sind, die nicht unbedingt ein gemeinsames Textziel anstreben. (Schwitalla 2006: 192) Was gesprochensprachliche und geschriebensprachliche Texte verbindet, sind nach Schwitalla (2006) bestimmte Bedingungen, die in den vorliegenden Daten erfüllte sind: Dabei geht es (1) darum, dass eine thematische Struktur erkennbar sein muss, die kohäsiv verbunden ist. (2) Zusätzlich muss eine handlungslogische Abfolge erkennbar sein. (3) Darüber hinaus unterliegen Texte einer bestimmen Funktion und werden (4) von einem Anfang und einem Ende begrenzt. (Schwitalla 2006: 192 f.) Für das vorliegende Projekt folgt daraus, dass mündliche Realisierungen ebenfalls als Texte erachtet werden. In den Daten treten sie entweder als eigenständige Erzähleinheiten auf oder sie sind in eine schriftliche Erzählung (z. B. als audiovisuelles Zitat oder personalisierte Binnenerzählung) integriert. Die Situation macht zugleich plausibel, dass für eine adäquate Phänomenbeschreibung der vorgefundenen narrativen Praktiken Erkenntnisse aus der mündlichen Erzählforschung zielführend sind. Dabei bleibt jedoch zu beachten, dass die mündlich realisierten Erzählungen und Erzählausschnitte mit wenigen Ausnahmen einem vorab geplanten Konzept entstammen, welches im Endprodukt mündlich vorgetragen wird. Es handelt sich somit keinesfalls um natürliche mündliche Erzählungen, die sich spontan entwickeln, auch wenn z. B. anhand „natürlicher“ Erzähler/innen der Anschein erweckt werden soll (siehe Abschn. 7.2.2.1). 
In Anbetracht des medialen Wandels wird nun zunehmend die „Forderung nach einem semiotisch erweiterten Textbegriff“ (Adamzik 2016: 60, vgl. auch Fix 2008a: 31 u. a.) formuliert. Dieser soll ebenso dem Nicht-Sprachlichen besondere Aufmerksamkeit widmen und will ,das Gemeinsame speziell visuell wahrnehmbare Zeichen, nämlich von Schrift und Bild [hervorheben]“ (ebd. 2016: 60). Diesbezüglich machte Schmitz (2003) in seinem Aufsatz Blind für Bilder darauf aufmerksam, dass die Textlinguistik Bildern erst einmal nur marginal Aufmerksamkeit widmete (,Sprachwissenschaft ignoriert Bilder - warum auch nicht?“, Schmitz (2003: 2)). Mit der Begründung, dass Schrift und Bild in der modernen Kommunikation

„vielfältige Koalitionen ein[gehen], die alte Grenzen zwischen Text und Bild auflösen und neue Metamorphosen und Synergien zwischen beiden ermöglichen" (Schmitz 2005: 195),

fordert er Sprachwissenschaftler/innen auf, der Multimodalisierung Rechnung zu tragen. Mittlerweile herrscht Konsens darüber, dass ausschließlich schriftlich realisierte Texte nur noch selten vorkommen, da im Zusammenspiel mit neuen technischen Möglichkeiten zunehmend visuelle Elemente in Texte integriert sind. Neben Mimik und Gestik für die mündliche Kommunikation sind es in Schrifttexten vorwiegend Bilder, deren Einbezug unter den Bezeichnungen visuelle Wende, pictural turn oder iconic turn (Bateman/Kesper/Kuhn 2013: 7; Stöckl 2011: 7; Bucher 2011: 123; Steinseifer 2009: 326; Ehlich 2005: 51; Sachs-Hombach 2003) in die Fachdiskussion eingehen. ,[D]ie traditionelle textlinguistische Auffassung, nur sprachliche Gebilde seien Texte“ (Dürscheid 2007: 275), wurde im nächsten Schritt zugunsten der Perspektive auf Texte als multimodale Einheiten aufgeben. Dabei habe vor allem die kommunikationsorientierte Linguistik sich engagiert, ihren Analysegegenstand der Entwicklung anzupassen, insbesondere

„seit Gunther Kress und Theo von Leeuwen das „Bilderlesen“ (1996) propagierten und in ihrem Diktum ,all texts are multimodal“ (Kress/van Leeuwen 1998: 186) auf den Punkt gebracht haben, dass Kommunikation nie in nur einem Zeichensystem verläuft" (Stöckl 2011: 10).

Das Zusammenspiel von Text und Bild wird häufig dahingehend kommentiert, dass Bilder die Textkommunikation ergänzen oder einschränken (Nöth 2010: 492 ff.) ${ }^{1}$. Bucher (2011: 123) geht dabei einen Schritt weiter, denn er

\footnotetext{
${ }^{1}$ Schmitz (2003: 2 ff.) beschreibt unter der Überschrift Sprachwissenschaft ignoriert Bilder - warum auch nicht? ausführlich den Forschungsstand um die Jahrtausendwende
} 
sieht den Kern dieses Wandels darin, dass so neuartige Mischformen entstehen, die er als multimodale Kommunikationsformen bezeichnet, welche ihre kommunikative Funktion nur im Zusammenwirken von Design (Farbe, Schriftgrößen und -arten, Anordnung der Elemente u. a.), Grafiken, Piktogrammen und Bildern/Fotografien entwickeln ${ }^{2}$. Kritischen Überlegungen, ob Bilder im engeren Sinn überhaupt in den Bereich der Sprachwissenschaften fallen (da sie de facto grundsätzlich verschieden zu sprachlichen Zeichen sind) ${ }^{3}$, steht die Entwicklung der Bildlinguistik entgegen. Sie wirft der Sprachwissenschaft vor, zugunsten der Analyse verbaler Kommunikation, Texte aus ihrem multimodalen Kontext herausgerissen und somit nicht umfassend in den Blick genommen zu haben (Holly 2006: 135 f.). Die Bildlinguistik zielt darauf, dieses „Versäumen“ aufzuarbeiten, indem sie Muster in der bildlichen Zeichenverwendung ergründet sowie die ,semantischen, perzeptuell-kognitiven und pragmatischen Unterschiede und Gemeinsamkeiten der beiden Codesysteme Sprache und Bild" (Stöckl 2011: 10) herausarbeitet. Um entsprechende Handlungspotenziale und pragmatische Funktionen solcher Texte nachzuzeichnen, ist der linguistischen Teildisziplin daran gelegen, „möglichst viele Gebrauchsdomänen von Sprache-Bild-Kombinationen“ (Stöckl 2011: 11) zu erfassen: Sie strebt einen multimodalen Textbegriff an. Stöckel sieht die Bildlinguistik als noch jungen Forschungsansatz, der sich im Laufe der Zeit noch stärker konturieren wird. Derzeit betrachtet er die Bildlinguistik eher als eine spezifische Perspektive innerhalb der Text- und Medienlinguistik, mit deutlicher Tendenz zur Textlinguistik, wenn er „materielle Bilder als Texte und Teile von Texten“ (Stöckl 2004a: 45) betrachtet. Diese radikale Position

zu Text-Bild-Relationen in einschlägigen linguistischen Werken. Damit zeigt er, dass die Sprachwissenschaft ihrem Gegenstandsbereich entsprechend Sprache fokussierte (,Linguisten sind bilderblind“, ebd. 2003: 7). Siehe hierzu auch Holly (2013), der in seinem Aufsatz Textualität - Visualität den Verlauf des Verhältnisses von Text und Bild in der Textlinguistik ausführt.

${ }^{2}$ Auch Schmitz (2005: 199) spricht vor allem in Zusammenhang mit Text-Bild-Relationen von „,komplexeren und oft effizienteren neuen symbolischen Formen“.

${ }^{3}$ Schmitz (2011: 29 ff.) weist in Bezug auf textuelle und bildliche Sehflächen darauf hin, dass linguistische Termini und Methoden für diese Untersuchungen nicht ohne Modifikation, Weiter- oder gar Neuentwicklung anwendbar sind. Auch Dürscheid (2007: $270 \mathrm{ff}$.) fasst grundlegende Unterschiede von Text und Bild auf, die zeigen, dass es nicht ohne Weiteres möglich ist, Bilder in eine linguistische Untersuchung einzubinden. Gemeinsamkeiten bestehen in ihrer Ausführung aus der semiotischen Sicht, während aus linguistischer und zeichentheoretischer Sicht grundlegende Unterschiede bestehen. So sieht beispielsweise Sachs-Hombach (2003) in den grundlegenden Unterschieden zwischen Text und Bild eine Begründung dafür, Bilder nicht in der Textlinguistik zu verorten. 
führt zu Gegenpositionen, wie sie beispielsweise Ehlich (2005) vertritt. Er wendet sich gegen eine Textualisierung des Bildes (Ehlich 2005: 59, „Bilder sind keine Texte"). Die einzigen Parallelen von Text und Bild sieht er in der Visualität. Der Trugschluss Bilder als Texte zu deklarieren, beruht seiner Meinung nach zu großen Teilen auf der Metonymie, dass Bilder gelesen werden müssten. Dabei werden seines Erachtens jedoch sowohl Bildlichkeit wie auch Textualität verkannt. Seine Aussage stützt er auf die Dichotomie, dass Rezipient/innen Texte im Lesevorgang linear wahrnehmen, während sie Bilder holistisch erschließen ${ }^{4}$. Ihm schließt sich Dürscheid an und argumentiert weiter, dass

„nur Texte auf sprachlichen Zeichen basieren; statische und bewegte Bilder dagegen sind Zeichenmodalitäten, die auf nicht sprachliche Zeichen rekurrieren“" (Dürscheid 2011: 96).

Sie kommt daher zu dem Ergebnis: „Sprache-Bild-Geräusche Kombinationen als Texte zu bezeichnen, halte ich für irreführend“" (Dürscheid 2011: 97). Trotz verschiedener Positionen die man zu der Frage, ob Bilder Texte sind, einnehmen kann, ist Schmitz (2003) beizupflichten, wenn er resümiert, dass in einer Zeit, die sich ,durch sehr viel raffiniertere, exaktere und reichere Codes und Gesten als die des Alphabets" (Schmitz 2003: 13) auszeichnet, eine Linguistik, die diese Entwicklung negiert, schnell antiquiert sein wird. Dahinter steht das mit der pragmatischen Wende verbundene Interesse, nicht ausschließlich den Sprachgebrauch, sondern auch mögliche Arten von Kommunikation zu untersuchen. Diese Perspektive teilen auch jene, die eine Erweiterung des Textbegriffs auf das Audiovisuelle nicht für gerechtfertigt erachten, insofern sie zwischen Text und Kommunikat (bspw. Dürscheid 2011: 96; Adamzik 2004: 43, 2016: 69 f.) unterscheiden. Kommunikate sind demnach „Äußerungsformen, die auf Sprache basieren, aber auch andere Zeichenmodalitäten enthalten können“ (Dürscheid 2011: 96). Kommunikate können dabei sowohl monomedial (als Text, Bild u. a.) oder multimedial in verschiedenen Kombinationen auftreten. Somit schließt die Unterscheidung von Text und Kommunikat nicht aus, nicht-schriftliche Zeichen (z. B. Bild, Grafik, Animation, Geste u. a.) in Verbindung mit Texten zu untersuchen. Den Terminus ,Text' behält Adamzik dabei jedoch ausschließlich dem schriftlichen Zeichen vor (vgl. Adamzik 2016: 69). Der Ansatz lässt eine weite Sichtweise zu, die also weitere Zeichen inkludiert, ohne jedoch den

\footnotetext{
${ }^{4}$ An dieser Stelle sei kurz auf das Linearitätsdogma (Krämer 2003: 159, in Dürscheid 2016: 212) verwiesen. Demnach ist die klassische linguistische Perspektive, Texte seien lineare Verkettungen, überholt. Es habe sich gezeigt, dass Texte räumliche und hierarchisch vielfach strukturierte Gebilde sind und dass auch Rezipient/innen Texte nicht nur linear lesen.
} 
Textbegriff zu erweitern ${ }^{5}$. Ich schließe mich hier Stöckl (2004a) insofern an, als das ich Bildern in den untersuchten Erzählungen eine kommunikative Funktion zuspreche. Für Klemm ist bspw. das Textualitätskriterium ,kommunikative Funktion“ und der damit verbundene soziale Sinn das ,am wenigsten verzichtbare Kriterium“ (Klemm 2002: 4) für Textualität. Denkt man an die Erhebungsplattformen Facebook, YouTube, Weblogs oder Twitter, ist der Einbezug von Bildern/Bewegtbildern für die Datenanalyse von Erzählungen unerlässlich. In den untersuchten Daten wirken insbesondere Fotografien an den Erzählungen mit. Sie benötigen aber dennoch das sprachliche Zeichen, das sie in der Erzählung situiert. Daraus folgt für das Korpus: Eine einzelne Fotografie ist für sich betrachtet noch nicht narrativ. Erst eine Serie von mindestens zwei aufeinander bezogenen Fotografien veranschaulicht in den erhobenen Erzählungen bspw. eine Zustandsveränderung. Die Fotografien treten in den Daten für gewöhnlich im Verbund mit schriftlichen Zeichen auf. Vor diesem Hintergrund sind Bilder meines Erachtens Elemente der Erzähltexte, denen situativ auch eine bestimme narrative Funktion zukommt (vgl. 9.3.4.2 und 9.4.5). Daher beziehe ich sie als unverzichtbaren Teil der Erzählungen in die Untersuchung ein.

Neben Text-Bild-Relationen von statischen Bildern stellt sich die Bildlinguistik auch in Ansätzen der Herausforderung, Bewegtbilder in die Untersuchungen einzubeziehen. In Anwendung von Konzepten der Zeichenhaftigkeit auf den Film merken Bateman et al. (2013: 9) kritisch an, dass semiotische und linguistische Ansätze zum Film meist noch mit überholten textlinguistischen Konzepten aus den 1980er Jahren arbeiteten ${ }^{6}$, die auf einem statischen Codebegriff gründen, der mit der heutigen Textauffassung nur schwerlich in Einklang zu bringen ist. Wildfeuer (2013) unternimmt den Versuch, auf die spezifischen Eigenschaften (Semiotizität, Multimodalität, Linearität, Situationalität, Intertextualität, Dynamizität, Temporalität, ebd. 2013: 42) von Filmen bezogen, eine sprachwissenschaftliche Definition für den Film als Text zu formulieren:

\footnotetext{
${ }^{5}$ Zum gegenwärtigen Zeitpunkt existieren bereits fundierte Arbeiten, deren Erkenntnisse für dieses Projekt gewinnbringend sind: Im wissenschaftlichen Diskurs werden Text-BildRelationen in verschiedenen text- und medienlinguistischen Arbeiten (z. B. Stöckl 2004a, 2008, 2011; Schmitz 2005; Diekmannshenke/Klemm/Stöckl 2011) untersucht. Darüber hinaus liegen Sammelbände vor, die verschiedene methodische Herangehensweisen an solche komplexen Texte veranschaulichen, wie der von Schneider und Stöckl (2011) Medientheorien und Multimodalität: Ein TV-Werbespot - Sieben methodische Beschreibungsansätze.

${ }^{6}$ Bspw. das Sender-Empfänger-Kommunikationsmodell, in dem der daraus abgeleitete Zeichen- und Codebegriff auch in der Textlinguistik als problematisch erachtet wird.
} 
„Der Film ist ein multimodales, das heißt mit sprachlichen und nicht-sprachlichen Zeichenmodalitäten sinnvoll strukturiertes und dynamisches, aber formal begrenztes Artefakt in zeitlich-linearer Abfolge. Er kann auf verschiedensten Ebenen intertextuelle Bezüge zu anderen Textvorkommen herstellen und je nach Kontext bestimmte Kommunikationsabsichten produzieren.“ (Wildfeuer 2013: 47)

Der Definition nach fasst Wildfeuer Filme als komplexe Texte, die nicht nur multimodale Aspekte beinhalten, sondern auch die linguistischen Kriterien der Linearität $^{7}$, Zeichenhaftigkeit ${ }^{8}$ und der kommunikativen Funktion ${ }^{9}$ aufweisen. Diese Option ist daher für das Projekt wichtig, da sich während der Datenerhebung zeigte, dass auch Bewegtbilder in Erzählungen von Unternehmen eingebunden sind oder gleich eine eigene Erzählung begründen (z. B. in Abschn. 9.1.2.2 und 9.4.5.2). Besondere Beachtung muss dabei dem Merkmal der Inszeniertheit solcher Filme geschenkt werden, die Holly (2004: 125) als sekundäre Audiovisualität bezeichnet. Damit drückt er aus, dass diese Formate von der natürlichen Kommunikation weit entfernt sind, da sowohl der Ton als auch die Bewegtbilder zugunsten der Inszenierung bearbeitet sind. Basierend auf einem „Rausch der Sinne“ (ebd. 2004: 125) durch die Komposition von Farben, Tönen, Bildern und Worten wird eine besondere Empfindungsintensität - eine sinnliche Vergegenwärtigung - angestrebt. Huth (1985) hat für das visuelle Material in Fernsehnachrichten 12 pragmatisch-semantische Funktionen ausgemacht, von denen für die Frage, inwiefern statische und bewegte Bilder einem narrativen Text zuzurechnen sind, folgende vier relevant sind:

1. „Rollenzuweisung für das abgebildete Medienpersonal

2.- 4. [ [...]

5. Demonstration des Inhalts verbaler Aussagen

6. Erläuterung der verbalen Aussagen

7.- 8. [...]

9. Dramatisierung der verbal dargestellten Ereignisse“ (Holly 2004: 129).

7 „Texte sind sinnvolle Verknüpfungen (sprachlicher) Zeichen in zeitlich-linearer Abfolge“ (Weinrich, 2005: 17, zit. nach Wildfeuer 2013: 43).

8 ,Semiotisch betrachtet können Texte definiert werden als nach Regeln geordnete Teilmengen (Reihen) sprachlicher Zeichen ..." (Breuer 1974: 25).

9 „Der Text muß immer als eine kommunikative Einheit, d. h. als eine thematische Einheit, die im Kommunika-tionsprozeß eine illokutive Funktion erfüllt, betrachtet werden“ (Rosengren 1980: 279). 
Innerhalb dieser Bereiche übernehmen Bewegtbilder Aufgaben, die Bestandteile der narrativen Themenentfaltung sind und sein können: zu 1) Einführung/Beschreibung von an der Handlung Beteiligten; zu 5 und 6) verdeutlichen, ausführen und spezifizieren von Aussagen; zu 9) das Dargestellte als essenzieller Teil der Spannungskurve, z. B. der Höhepunktgestaltung. Dennoch ist die Domäne des Bildes vorrangig das Sehen (Holly 2006: 147), womit das Merkmal der semantischen Unbestimmtheit einhergeht (Sachs-Hombach 2003: 174 ff., nach Holly 2006: 143). Mit Blick auf die erhobenen Erzählungen, ist das sprachliche Zeichen gewichtig, denn es bringt die Bilder und Bewegtbilder in der Geschichte im Sinne der (S)D erst in den erforderlichen Gesamtzusammenhang. Allerdings deutet sich in dem spezifischen Zusammenspiel von Text und Bild auch an, dass die Bilder für die gesamte Erzählung konstitutiv und somit funktionsorientiert sind - und zwar hinsichtlich einzelner Elemente einer Geschichte und damit auch für die gesamte Erzählung (vgl. Große (2011: 18 ff.) zur funktionsorientierten Bild-Linguistik). Für die erhobenen Daten ist zur Vermittlung einer Geschichte demnach die Sprachlichkeit maßgebend. Da in den vorliegenden Erzählungen jedoch die sprachlichen Zeichen sehr eng mit weiteren semiotischen Ressourcen in Bezug auf die Geschichte verbunden sind, referiere ich auf den multimodalen Verbund im Weiteren als multimodale Realisierungen in Form von multimodalen Erzähltexten. Vor diesem Hintergrund betreffen die nun folgenden allgemeinen Charakteristika das kommunikative Gesamtprodukt, also die multimodalen Erzähltexte.

\subsection{Klassifizierung der Daten als ,Repräsentationstexte}

Ich leite zuerst mit grundsätzlichen, textlinguistischen Merkmalen ein, um die erhobenen Daten im weiteren Verlauf sukzessive zu spezifizieren, indem ich sie letztlich als Variante unter eine spezifische Textsorte der institutionellen Wirtschaftskommunikation summiere.

Unter einer kommunikativ-pragmatischen Perspektive obliegt den Erzählungen die Aufgabe, zwischen den Unternehmen und ihrer Umwelt zu vermitteln (Imageaufbau). Damit ist ihr Handlungsbereich auf Ausschnitte aus der jeweiligen Unternehmenswelt bezogen (speziell hierzu, Abschn. 7.2.1). Durch die Publikation der Erzählungen auf verschiedenen Internetplattformen ist der Handlungsbereich zudem öffentlich. Das Herstellen von Öffentlichkeit auf den ausgewählten Internetplattformen (Kommunikationsmedien) wirkt sich wiederum auf 
die zeitliche Dimension der Kommunikation aus, insofern der Erzähl- und Rezeptionsvorgang nicht synchronisiert sind ${ }^{10}$. Dabei ist die Handlungsrichtung der Texte primär nach außen gerichtet (interne vs. externe Kommunikationsrichtung, Gohr 2002: 135). Aufgrund der Vermittlungsaufgabe und der über das Internet hergestellten Öffentlichkeit sind die Erzählungen ,inhärent mehrfachadressiert“ (Burel 2015: 153). Damit geht einher, dass die Erzählungen polyfunktional (vgl. ebd.) sind. Mögliche Funktionen ${ }^{11}$ dieser Erzählungen sind neben der - meiner These nach dominanten - Funktion der Imageförderung die Optionen sowohl als Informations- und Identifikationsangebot für Unternehmensmitglieder wie auch gleichzeitig der Kontaktaufnahme und Beziehungskonstitution mit den Bezugsgruppen zu dienen. Sowohl in Blogbeiträgen, Tweets und Facebook Posts finden sich vereinzelt Begrüßungs- (z. B. Hallo) und Abschiedsformeln (z. B. Eure/r, bis bald, u. a.) oder Handlungsaufforderungen (z. B. in der Art wenn Ihr Euch auch für ... interessiert, dann folgt uns auf ... / folgt dem Link oder schreibt uns, was ihr denkt / wie es Euch ergangen ist). Allerdings ist durch die Formulierungen die Adressierung unspezifisch, um den Adressatenkreis möglichst offen zu halten (vgl. hierzu auch Tophinke 2009: 268). Folgt aufgrund einer solchen Handlungsaufforderung eine Rezipientenreaktion, dann sind die Abstände zeitlich stark zerdehnt oder die Reaktion ist nicht auf die Aufforderung bezogen. Das erweckt den Eindruck einer im Grunde monologischen Konzeption, deren Aufgabe darin besteht, in erster Linie eine Mitteilung/Botschaft zu platzieren. Selbst diejenigen Erzähler/innen, die zum Dialog auffordern, erhalten nur eine geringe Resonanz (siehe z. B. Abschn. 9.2.1). Des Weiteren begünstigt die digitale Erzählumgebung ${ }^{12}$ eine narrative Umsetzung anhand unterschiedlicher Zeichensysteme ${ }^{13}$. Die grundlegenden Zeichensysteme in den gesammelten Daten beruhen auf gesprochener und geschriebener Sprache. Sie sind wiederum mit weiteren auditiven (Geräusche, Musik) und visuellen (Fotografien, Abbildungen, typografische Gestaltung, Bewegtbilder) Zeichen in unterschiedlicher Funktion kombiniert. Dadurch sind die Erzählungen multimodal angelegt (äußere Textgestaltung) und können ggf. transmedial veröffentlicht sein (Veröffentlichung einer

${ }^{10}$ Vgl. Dürscheid (2003: 10) zur zeitlichen Dimension der Kommunikation: Zerdehnen der Sprechsituation.

${ }^{11}$ Also der „Zweck [...], den der Produzent mit dem Text zu erreichen sucht“ (Adamzik 2016: 171).

${ }^{12}$ Zur Erzählumgebung (narrative environment) siehe Gubrium/Holstein (2008: 247 f.).

${ }^{13}$ Ich folge der allgemein anerkannten Betrachtung von Holly, der Medien als ,materielle Hilfsmittel“ (Holly 1997: 69 f.) auffasst, ,,mit denen Zeichen verstärkt, hergestellt, gespeichert und/oder übertragen werden können“ (ebd.). Medien ermöglichen dadurch eine Kommunikation auf Distanz. 
Geschichte auf unterschiedlichen Internetplattformen). Der Herstellungsaufwand für die Erzählungen variiert stark, denn er hängt davon ab, welche Akteure (z. B. professionelle Kommunikationsagenturen, Autorenkollektive, Mitarbeiter/innen) daran beteiligt sind und wie aufwendig die Geschichte umgesetzt ist (Text, Bild, Animation, Film u. a.).

Möchte man die Merkmale der Daten weiter fassen, kann dies unter Zuhilfenahme von Textsorten geschehen. Dabei handelt es sich um ,historisch gewordene, konventionalisierte, normhaft wirkende Muster sprachlichen Handelns“ (Heinemann 2000: 516). Daraus folgt, dass in unserem Alltagsverständnis mit Textsorten konkrete Textexemplare verbunden sind (ebd.). Bei Textsorten handelt es sich um

„Einheiten auf relativ niedriger Abstraktionsebene, die außer durch den Kommunikationsbereich und andere situative Faktoren v.a. auch als funktional spezifiziert verstanden werden“ (Adamzik 2016: 128).

Eine solche Zuordnung ,trägt der Verankerung der Textsorten in übergeordneten Handlungszusammenhängen Rechnung“ (Brinker et al. 2000: XIX f., nach ebd.). Die Spiegelung, inwiefern die vorliegenden Daten gemeinsame und atypische Merkmale mit Textsortenklassifizierungen aufweisen, charakterisiert die Erzählungen weiter und grenzt sie zugleich auf einen Bereich ein.

Die erhobenen Daten sind Bestandteil der übergeordneten Textsorte ,Gebrauchstexte'. Unter Gebrauchstexten werden grundsätzlich Texte ${ }^{14}$ verstanden, ,mit denen üblicherweise kein besonderer ästhetisch-literarischer Anspruch erhoben wird“" (Dimter 1981: 35). Diese Unterscheidung basiert auf dem Vergleich mit literarischen Texten. So hält auch Belke (1975) fest, dass es sich bei Gebrauchstexten um Texte handelt,

„die nicht, wie poetische Texte, ihren Gegenstand selbst konstituieren, sondern die primär durch außerhalb ihrer selbst liegende Zwecke bestimmt werden. Gebrauchstexte dienen der Sache, von der sie handeln; sie sind auf einen bestimmten Rezipientenkreis ausgerichtet und wollen informieren, belehren, unterhalten, kritisieren, überzeugen, überreden oder agitieren“ (Belke 1975: 320, Hervorhebung, U.A.).

Dabei ist die Grenzziehung zwischen literarischen Textsorten und Gebrauchstexten nicht immer trennscharf möglich. Insbesondere, da literarische Texte in

\footnotetext{
${ }^{14}$ Vielfach wird der Begriff ,Gebrauchstext" für Schriftkommunikation verwendet (siehe Rolf 1993). Aus dem Bereich ausgegrenzt werden insbesondere Gespräche. Für sie wird meist analog der Terminus ,Gesprächssorte“ verwendet. (Heinemann 2000: $514 \mathrm{f}$.)
} 
der Unterscheidung zu Gebrauchstexten mit fiktionalen Texten gleichgesetzt werden (Adamzik 2016: 117). Das betrifft mitunter ebenfalls den Unternehmensbereich, da im Zuge der wachsenden Publikumsorientierung und dem zunehmenden Unterhaltungsbedürfnis Entgrenzungsprozesse beobachtbar sind (Krüger 2015: 108) ${ }^{15}$. Ein weiteres Differenzierungskriterium ist nach Harweg (1979: 327), dass ein literarischer Text für ein Publikum geschrieben sei, ein Gebrauchstext dagegen nicht. Auch dieses Kriterium ist für die vorliegenden Daten mit Blick auf das Ausschlusskriterium ,Publikum` einzuschränken. Insbesondere die inhaltliche Gestaltung (Fokus auf das Erlebnis: Party, Freizeit, Gemeinschaft) aber auch sprachliche Merkmale (wenig Fachlexik, eher umgangssprachliche Lexik) deutet darauf hin, dass Unternehmen ihre Veröffentlichungen an ein antizipiertes Zielpublikum anpassen. Doch trotz der unklaren Grenzen, würde niemand diese Erzählungen als literarisch bewerten ${ }^{16}$. Als uneingeschränkt zutreffend sehe ich die Aussage von Rolf (1993), der zufolge Gebrauchstexte „so etwas wie einen Funktionswert, literarische Texte aber [...] einen Eigenwert" (Rolf 1993: 128) haben. Unternehmen investieren keine Zeit und kein Geld in Erzählungen um ihrer selbst willen. Von der Veröffentlichung erwarten Unternehmen dementsprechend einen (langfristigen monetären) Mehrwert ${ }^{17}$. Aufgrund dieser Eigenschaften können die Daten nach der Klassifikation von Belke am treffendsten den ,publizistischen Gebrauchstexte[n]“ (Belke 1975: 324, nach Gohr 2002: 304) zugerechnet werden. Sie beinhalten Bericht, Reportage, Glosse, Anzeige und Werbetext. Betrachtet man die Textsorte ,Gebrauchstexte" als einen übergeordneten Begriff für verschiedene Textsortenvarianten, lässt sich das Datenmaterial zunehmend spezifischeren Differenzierungskriterien zur Klassifizierung zuordnen.

Es besteht eine Vielzahl an Klassifikationsansätzen für Textsorten ${ }^{18}$. Aus diesem Grund beschränke ich mich auf Beispiele, die auf wirtschaftssprachliche Textsorten ausgerichtet sind. In diesem Zusammenhang bezieht sich ein naheliegendes Differenzierungskriterium auf die Ebene des Vorkommensbereiches ,Wirtschaft‘. Darauf verweist bspw. die Rückbindung an das Unternehmen als Textproduzent, in dem Kompositum ,Unternehmenstexte“ (Diatlova 2003):

\footnotetext{
${ }^{15}$ Für weitere Argumentationen vgl. Rolf 1993: 125 ff.; Burger 2014: 129; Brinker et al. 2014: 20, 134 f.; Adamzik 2016: 115 f. u. a.

${ }^{16}$ Insbesondere in Bezug auf Poetizität und bedingt im Falle einer Ästhetisierung.

${ }^{17}$ Man denke an das Sozialkapital, Abschnitt. 2.1.

${ }^{18}$ Einen differenzierten Überblick bietet Rolf (1993: 81 ff.). Er erörtert ganze 23 Klassifizierungsvorschläge, bevor er seinen eigenen funktionalen Ansatz unterbreitet.
} 
„Der Unternehmenstext ist Ausschnitt aus dem Unternehmensdiskurs, der als Kommunikations- bzw. Handlungsmittel der Unternehmenskommunikation im Rahmen bestimmter Unternehmensaktivitäten/-schritte und $\mathrm{zu}$ einem bestimmten betrieblichen Zweck als zusammenhängend emittiert und in sich abgeschlossen deklariert wird.“ (Diatlova 2003: 19)

Der Untersuchungsgegenstand, auf den sich die Autorin bezieht, betrifft die Textsorten Anfrage, Bestellung, Präsentation einer Firma/eines Unternehmens, Mitteilung, Angebot, Vertrag, Protokoll, Industrieller Entwicklungsplan, Geschäftsbericht und die Pressemitteilung als „Instrumente für die Durchführung der wichtigsten Unternehmensaktivitäten (ebd. 2003: 98). Innerhalb dieser Textsorten kommt die Textsorte ,Präsentation eines Unternehmens' den Daten am nächsten. Das Definitionskriterium ,in sich geschlossen' ist jedoch für die Daten von Twitter im Normalfall nicht zutreffend. Zutreffend für die Daten ist in dieser Definition, dass es sich um einen Ausschnitt aus dem Unternehmensdiskurs handelt. Dabei ist jedoch zu beachten, dass es sich um einen initiierten, gemachten Diskurs handelt, der der Außendarstellung dient. Die Zweckorientierung belegt jedoch, dass die Erzählungen ein Handlungsmittel der Unternehmenskommunikation darstellen. Unabhängig von der Definition geht die Autorin davon aus, dass Textsorten Problemlösungsmuster bereitstellen. Das bedeutet, dass die Textsorte einem Textmuster unterworfen ist, das dem/der Textproduzent/in und rezipient/in auf der Textebene als eine „kommunikative[] Routine“ (Adamzik 1995a: 28, nach Hundt 2000: 642) vertraut ist. Diese Annahme ist für die vorliegenden Daten nicht immer zutreffend. Erzählungen und Erzählfragmente auf Facebook und Twitter zeigen, dass die Erzähler/innen noch in einer Orientierungsphase sind. Das reflektieren die Verfasser/innen durchaus auch selbst, wenn - wie in diesem Beispiel der Dt. Telekom - mit den Worten eingeleitet wird: „wir versuchen heute mal ein anderes Format" (Dt. Telekom, Twitter, o. J. Korpusquelle: DTKOM_809_WB) und der Autor das Vorgehen anschließend im Netz reflektiert. Das verdeutlicht, dass auf bestimmten Internetplattformen solche Routinen noch in der Entwicklung sind. Bezieht man die Textsortenklassifizierung ausschließlich auf den Vorkommensbereich - also den Weltausschnitt, auf den die Textsorte fokussiert -, mahnt Hundt (2000: 642) an, dass die Eingrenzung unzureichend ist (vgl. auch Gohr 2002: 89). Er sieht bei diesem Vorgehen ein Abgrenzungsproblem, da der Kommunikationsbereich wirtschaftsbezogener Textsorten mit anderen Bereichen (z. B. Verwaltung, Rechtswesen, Politik) verflochten ist (Hundt 2000: 644). Andere Klassifizierungsoptionen finden sich bspw. in Form von prototypischen Konzeptionen, wie ,Leitbild und leitbildähnlichen Dokumenten“" (Stach/Held 2009: 7, nach Burel 2015: 149, Hervorhebung i. O.) 
oder ziehen inhaltliche Spezifikatoren hinzu, wie bspw. Ebert (2001) im Fall von unternehmenspolitischen Texten oder unternehmensphilosophischen Texten. $\mathrm{Zu}$ diesen Texten zählt der Autor u. a. die Unternehmensgeschichte, aber auch Unternehmensziele, -werte oder das Leitbild. (Burel 2015: 149)

Eine weitere Klassifikationsoption ist die Bezugnahme auf die Textfunktion. Darunter fallen bspw. Vermittlungstexte (Hundt 2000: 654, Felder 2009: 44), deren dominante Funktion - wie die Bezeichnung nahelegt - der Wissenstransfer ist. Hundt macht die Kategorisierung vermittlungssprachlicher Textsorten für den Bereich der Wirtschaft fruchtbar, indem er sich darauf bezieht, dass Vermittlungssprache für Kommunikationsformen konstitutiv ist, die zwischen Bezugswelten vermittelt (Hundt 2000: 654). Das bedeutet eine „Vermittlung zwischen Institutionen und Alltag" (ebd. 2000: 642). Als Beispiele für bestimmte Textebenen nennt Hundt Pressetexte (Leitartikel, Kommentar), Werbung oder Kundeninformationen. Aufgrund der Funktion, etwas zu vermitteln, sind die Texte an ihren Adressat/innen orientiert. Einen Sonderfall stellt die Werbung da. Sie geht über die vermittelnde Funktion hinaus, indem ihr zusätzlich eine direktive Textfunktion zukommt. Nach den zuvor erläuterten Kriterien für ,Unternehmenstexte', die nur mit Einschränkungen zutreffen, ließen sich die erhobenen Daten durchaus als eine Textsortenvariante unter vermittlungssprachlichen Textsorten einsortieren, da es um die Vermittlung eines ,konstruierten Selbstbild[es]“ (Burel 2015: 150) geht. Dennoch bietet Gohr (2002) einen noch passenderen Ansatz für das vorliegende Korpus an, dem ich mich nun anschließe $\mathrm{e}^{19}$.

Gohr (2002) analysiert in ihrer Dissertation 140 Geschäftsberichte und Briefe an Aktionäre mit dem Ziel einer Textsortenbestimmung. Im Ergebnis ordnet sie die Daten unter die publizistischen Gebrauchstexte (Belke 1975) in die Gruppe ,Repräsentationstexte' ein. Repräsentationstexten spricht sie ein übergeordnetes Prinzip zu: „Sie stehen im Dienst des Unternehmens und haben die Aufgabe, dieses optimal zu repräsentieren" (Gohr 2002: 304). Die Terminologie hat sozusagen die (S)D in Form der funktionalen Ausrichtung ,Repräsentation-" bereits in ihrem Namen. Burel (2015: 150) erachtet die Bezeichnung darüber hinaus gerade für identitätskonstituierende Texte als zutreffend, da durch das Lexem ,repräsentieren " deutlich wird, dass die kommunizierte Wirklichkeit durch sprachliche Zeichen vermittelt ist. Das schließt zugleich den konstruierenden Aspekt der (S)D von Unternehmen mit ein. Aus diesem Grunde verorte ich die Daten unter Repräsentationstexten. Dabei schlage ich vor, die Erzählungen als eine Variante

\footnotetext{
${ }^{19}$ Vgl. ebenfalls Burel (2015: 149 f.) im Fall von Identitätspositionierungen der DAX-30Unternehmen.
} 
von Repräsentationstexten anhand des Arbeitsbegriffs ,Repräsentationserzählungen' aufzunehmen. Mit dem terminologischen Eingriff verweise ich nicht nur auf die thematische Entfaltung innerhalb der Daten, sondern nehme auch den Fokus von einer Eingrenzung auf schriftliche und mündliche Zeichen. So ist die multimodale Realisierung der Daten in der Bezeichnung berücksichtigt. Des Weiteren stelle ich dem Terminus Repräsentationserzählungen mit der Spezifizierung unternehmensbezogene Repräsentationserzählungen (uRE) den Hinweis auf ihre Bezugswelt zur Seite. Die Spezifizierung erachte ich als sinnvoll, da die ermittelten narrativen Praktiken trotz Überschneidungen nicht mit narrativen Praktiken von Privatpersonen gleichzusetzen sind. Nichtsdestotrotz handelt es sich bei den Daten um eine Variante der Textsorte ,Repräsentationstexte'.

Das Ziel der Ausführung war es, auf spezifische Eigenschaften der Erzählungen zu verweisen, um die erhobenen Daten im Fachdiskurs zu verorten. Es ist somit nicht intendiert eine Textsorte vorzuschlagen, sondern lediglich einen Arbeitsbegriff herauszufiltern, der die Daten angemessen beschreibt.

\subsection{Gemeinsamkeiten und Unterschiede zur Werbung}

Sich positiv darzustellen, ist bereits ein Versuch der Beeinflussung, was charakteristisch für das Werben an sich ist (vgl. Janich 2013: 18). Allerdings geht es hier um ein Werben für das Unternehmen als Ganzes ${ }^{20}$. Hierbei steht nicht das Produkt oder die Dienstleistung im Mittelpunkt, sondern Immaterielles (Einstellungen, Werte, Unternehmensethos oder -moral). Das Werbeobjekt ist somit das, wofür das Gesamtunternehmen stehen möchte. Als Werbung definiert Hoffmann (1981) grundsätzlich

„die geplante, öffentliche Übermittlung von Nachrichten [...], wenn die Nachricht das Urteil und/oder Handeln bestimmter Gruppen beeinflussen und damit einer Güter, Leistungen oder Ideen produzierenden oder absetzenden Gruppe oder Institution (vergrößernd, erhaltend oder bei der Verwirklichung ihrer Aufgabe) dienen soll." (Hoffmann 1981: 10, nach Janich 2013: 19)

Da uRE das übergreifende Ziel verfolgen, das Unternehmensimage aufzuwerten, indem sie mithilfe spezifischer (S)D das Unternehmen als sympathisch und attraktiv für bestimmte Bezugsgruppen charakterisieren, dienen die Erzählungen dem Eigenzweck in Form des Erhalts des Unternehmens und der Umsetzung

${ }^{20}$ Firmenwerbung als ein Bereich der Public Relations, siehe hierzu Abschnitt. 7.1.1. 
unternehmensspezifischer Aufgaben. Auf dieser Basis stellen die in die Erzählungen eingearbeiteten (S)D einen Versuch von Unternehmen dar, ihre Umwelt in einem nutzenmaximierenden Sinne zu beeinflussen. Dadurch besteht eine enge Verbindung zu dem, was wir mit der „klassischen“ Werbung von Wirtschaftsunternehmen gleichsetzen: die Produktwerbung. Für eine Spezifizierung des Untersuchungsgegenstandes ist es daher erforderlich, sich damit auseinander zusetzen, worin die erhobenen Daten Parallelen und Unterschiede zur „klassischen“ Werbung aufweisen.

Sowohl die Produktwerbung wie auch uRE streben eine originelle Umsetzung an, um den Rezipient/innen im Gedächtnis zu bleiben und wiedererkannt zu werden. In den uRE spiegelt sich das z. B. in Form von animierten Bewegtbildern, Filmsequenzen und auditiven Einschüben in Unternehmensgeschichten sowie einer interaktiven Nutzeroberfläche mit verspielten Elementen. Ebenso beziehen Unternehmen ,attraktive“ Internetplattformen (Facebook, Twitter, YouTube) ein und experimentieren hierbei mit dem Erzählen selbst (z. B. diskontinuierliche Erzählungen auf Twitter, Mitarbeitererzählungen auf Corporate Blogs). Dabei ist für beide Werbebereiche gleichermaßen von Bedeutung, dass die Botschaft variierend wiederholt wird (vgl. Adamzik 2012: 24). In den uRE geschieht das einerseits durch die Wiederholungen einer spezifischen (S)D durch verschiedene Sprecher/innen oder aufgrund von Reformulierungen der (S)D auf verschiedenen Internetplattformen ( $=$ transmedial), jedoch mit dem selben Erzählthema.

Allgemein werden Werbetexte den appellativen Texten zugerechnet: Ein „Emittent versucht, den Rezipienten dazu zu bringen, ein bestimmtes Produkt zu kaufen“ (Brinker et al. 2014: 113). In diesem Punkt besteht eine Gemeinsamkeit und ein maßgebender Unterschied zwischen uRE und der Produktwerbung. Die Gemeinsamkeit ist darin zu sehen, dass auch in den uRE etwas ,verkauft“ werden soll, nämlich das positive Image. Darin liegt allerdings auch ein grundlegender Unterschied: Es handelt sich nicht um ein Produkt, sondern um eine behauptete „Wahrheit“" bzw. „Wirklichkeit“ des Unternehmens. Gemeinsam ist beiden Bereichen, dass die Kauf-, oder im Falle von uRE, die Glaubensaufforderung selten durch performative Formeln explizit gemacht werden. Sowohl in Werbetexten wie auch in uRE wird häufig vermittelt kommuniziert, indem eine Gewährsfrau oder ein Gewährsmann auftritt, die/der das Produkt bzw. das Unternehmen empfiehlt. Vorrangig geschieht das anhand von Einstellungsbekundungen und Informationsvermittlung. In der klassischen Werbung werden hierfür gerne Autoritätspersonen ins Feld geführt, während es in den uRE vorrangig unternehmensexterne Personen, Mitarbeiter/innen oder U.-Gründer sind (siehe 
Abschn. 7.2.2). Eine weitere Gemeinsamkeit liegt darin, die Rezipient/innen emotional anzusprechen, um ihre Einstellung zu beeinflussen ${ }^{21}$. Allerdings basiert die Beeinflussung auf unterschiedlichen Verfahren der thematischen Entfaltung. Während Werbetexte argumentativ verfahren (vgl. Brinker et al. 2014: 113 f.), entwickeln sich die vorliegenden Daten narrativ. Diesbezüglich sind uRE so angelegt, dass die selbstdarstellenden Merkmale zum bestimmenden Hintergrund und zur Triebfeder für die Geschichte werden. Das bedeutet, die (S)D bedingt die Auswahl der Ereignisse und die Aufbereitung der narrativen Themenentfaltung. So ist sie nicht nur unaufdringlich als Bestandteil der Geschichte legitimiert, sondern muss auch tatsächlich erfolgen.

Für die Werbung ist ein charakteristisches Set an Bausteinen kennzeichnend. Es handelt sich um abstrakte Kategorien, ,die durch ein Schema vorgegeben sind“ (Adamzik 2012: 138). Den Kern bilden „die Bausteine ,Benennen/Zeigen“, ,Beschreiben“ und ,Bewerten“" (ebd.). Diese Bausteine sind ebenfalls charakteristisch für uRE. Unterschiedlich ist hingegen, dass die Produktwerbung zwar realitätsnah agiert, aber den Verbraucher/innen in der Regel klar ist, „dass es sich um gestellte Szenen handelt, [...] es sich um klare Montage handelt oder die Bilder nicht-realistisch sind“ (Adamzik 2012: 140). In uRE wird dagegen darauf geachtet, dass bspw. die Fotografien möglichst wie Schnappschüsse wirken, um größtmögliche Authentizität zu suggerieren (vgl. z. B. Abschn. 9.3.4.1). Dadurch, dass der Referenzbereich auf eine bestimmte Unternehmens,,-wirklichkeit“" abzielt, würde sich eine nicht-realistische Darstellung von uRE äußerst ungünstig auf die (S)D der Unternehmen auswirken.

Dieser Aspekt stellt den maßgebenden Unterschied zwischen der Produktwerbung und uRE dar. Im Laufe der Zeit wurde die Produktwerbung zu einer Unterhaltungsform mit Kultstatus:

„Damit kann sie sich nicht nur von den zweckrationalen Handlungszusammenhängen ablösen, sondern ist auch in ihrer Darstellungsfunktion nicht mehr nur bezogen auf die reale Welt: Sie umfasst Aussagen, deren Wahrheitsgehalt schlechterdings nicht überprüft werden kann, und schafft fiktionale Welten.“ (Adamzik 2012: 128)

Das führt zu einer gemeinhin tolerierten Offenheit in Bezug auf Fiktionalität, die uRE in diesem Ausmaße nicht gestattet ist. Ein fiktionaler Text zeichnet

\footnotetext{
${ }^{21}$ Das Anreichern eines Textes mit Emotionen ist Mittel des Anpreisungsverfahrens, das als ein Kennzeichen eines werblichen Stils aufgefasst wird. Der werbliche Stil ist daran zu identifizieren, dass er eine ,persuasiv ausgerichtete Stellungnahme (Haltung/Einstellung) des Werbers zu seinem Kommunikationsgegenstand, d. h. zur beworbenen Sache [darstellt]“ (Hofmann 2012: 180).
} 
sich dadurch aus, dass er sich aus verschiedenen Akten zusammensetzt, wie dem Fingieren, der Selektion von verschiedenen Komponenten und deren innertextueller Kombination (Burger/Luginbühl 2014: 203). In den Literaturwissenschaften werden zudem „Fiktionssignale“ (Zipfel 2001: 232) diskutiert, die einen fiktionalen Text indizieren ${ }^{22}$. Tauchen solche Signale oder Indikatoren auf, ist den Rezipient/innen für gewöhnlich schnell bewusst, dass die Darstellung nicht „die“ Wirklichkeit abbildet. Dabei knüpft diese Wirklichkeit an ein vor-theoretisches, alltagssprachliches Konstrukt an, das auf Weltwissen basiert ${ }^{23}$. Im Bezugsbereich von Unternehmen ist eine fiktionale Erzählung der Norm nach unmissverständlich als solche gekennzeichnet. Gerade in der Werbung unter dem Einfluss des Storytelling können in einer fiktionalen Erzählung ebenfalls Realitätsfragmente vorkommen, wie z. B. in der Telekomwerbung mit der erfundenen Familie Heins. Die Werbung folgt dem sogenannte Fictionality Ansatz, indem in die Geschichte mit den erfundenen Figuren der Familie Heins - „reale“ Personen als Figuren eingearbeitet sind: Sie spielen sich selbst. Solche fiktionalen, werbenden und unterhaltenden Erzählungen unterscheiden sich in ihrem ontologischen Status von der Lüge und der Täuschung, da sie als fiktional kenntlich gemacht wer$\operatorname{den}^{24}$. So ist ihr Bezugsbereich auf eine erdachte Welt erkennbar und für die Glaubwürdigkeit der Unternehmen ungefährlich.

Mit dem Weltausschnitt auf den sich eine Erzählung bezieht, ist in der Linguistik das Kriterium des Realitätsbezugs verbunden. Also die Frage, inwieweit die Inhalte mit der Realität übereinstimmen (vgl. Adamzik 2008: 155, 2016: 115; Gohr 2002: 115; Burger 2014: 203). Es geht demnach um einen Wirklichkeitsbezug, dem Dimter (1981) ein naives Alltagsverständnis von Realität zugrunde legt, welches ,per definitionem menschliche, und das heißt immer auch gesellschaftlich vermittelte Realität [beinhaltet]“ (Dimter 1981: 101). Damit einher geht also

${ }^{22}$ Das trifft z. B. auf Erzählungen in der dritten Person zu, wenn Verben von inneren Vorgängen in Bezug auf Dritte auftauchen, die Erzähler- mit der Figurenperspektive vermengt wird oder ein/e Erzähler/in in großem Umfang sehr detailreich erzählt (Zipfel 2001: 235). Aus der linguistischen Perspektive werden solche Signale kritisch gesehen. Aufgrund dessen, dass sie auf gesellschaftlichen Konventionen beruhen, sind sie keine stabile Größe, sondern vielmehr variabel und zeitlich gebunden: ,There is no textual property, syntactical or semantic that will identify a text as a work of fiction“ (Searl 1975: 325, nach Saupe/Wiedemann 2015: 6 f.).

23 „Nach Goodman ist die Alltagswirklichkeit das, was den Mitgliedern einer Gesellschaft als wirklich oder real gilt. Das Wissen darum, was als wirklich gilt, setzt sich aus einem Wissen über verschiedene Teile unterschiedlicher Welt-Versionen zusammen. " (Zipfel 2001, 74 f., nach Burger/Luginbühl 2014: 203)

${ }^{24}$ Inwieweit die Rezipient/innen das erkennen, ist ein anderes Thema. Kommentare zur Familie Heines auf YouTube indizieren, dass es mitunter zu Verunsicherungen kommt. 
die Frage, ob die erzählten Inhalte ,real“" sind. Prüfbar ist das nur, wenn Referenzpunkt für die erzählten Ereignisse zum Erzählzeitpunkte für die Rezipient/innen in der außersprachlichen Welt verifizierbar sind. Dann

„heißt Wahrheit von Propositionen [...]: Für alle referentiellen Ausdrücke konnten Referenzpunkte in der realen Welt zum angegebenen Zeitpunkt aufgefunden werden, und alle Prädikationen über diese referentiellen Ausdrücke bilden Eigenschaften, Handlungen, Zusammenhänge usw. der Elemente der realen Welt zum angegebenen Zeitpunkt ab.“ (Dimter 1981: 101)

Ist das zutreffend, fasst Dimter das Merkmal unter dem Terminus ,faktizitätstreue“ (ebd.). Sind solche Referenzpunkte in der realen Welt nicht gegeben, bezeichnet er den Gegenstand als fiktional. Da die uRE uns vermitteln, auf reale Ereignisse, Handlungen und Gegebenheiten rund um die Unternehmen zu referieren, sind sie in diesem Bereich als faktizitätstreu einzustufen. Allerdings beinhaltet diese Eigenschaft noch nicht das narrationsspezifische Merkmal der „Erfahrungshaftigkeit“ (Fludernik 1996). Doch gerade die Art des persönlichen Erlebens macht den Unterschied zur Werbung aus, denn Referenzpunkte aus der realen Welt sind in Form der beworbenen Produkte auch in der Werbung anzutreffen. Im Falle von Meinungen und Bewertungen als Ausdruck des Erlebens kann hingegen schwerlich von Fakten gesprochen werden. Zwar ist in den uRE das Erleben immer an überprüfbare Ereignisse als Referenzpunkte rückgebunden $^{25}$, doch ist das eher als ein rhetorischer Akt zu begreifen, den sich auch die Produktwerbung zu eigenen macht. Um sozusagen Außersprachliches wie das Erleben als Spezifikum der uRE zu beschreiben, interpretiere ich Lux (1981) Definition darüber, inwiefern etwas real ist. Nach Lux ist das daran zu erkennen, dass ,der abgebildete Sachverhalt [...] zumindest vom Textproduzenten als ,wirklich“ gemeint [ist]“ (Lux 1981: 239, nach Gohr 2002: 150). Meine Interpretation des Zitats zielt darauf, dass der von der Textproduzentin oder vom Textproduzenten abgebildete Sachverhalt auch auf dessen Meinung oder Erfahrung angewendet werden kann. Handelt es sich bei dem Sachverhalt um Meinungen oder Erfahrungen, dann können sie insofern als real betrachtet werden, als der/die Textproduzent/in sie für sich als wahrhaftig deklariert (Zitatbezug: vom/von der Textproduzent/in als ,wirklich“ gemeint ist). Im Falle der uRE könnte man nach dieser Auslegung von einer personenbezogenen Realität ausgehen, die in Bezug auf persönliche Erfahrungen Wahrhaftigkeit impliziert. In Bezug auf die Werbung ist den Rezipient/innen für gewöhnlich bewusst, dass

${ }^{25}$ Bspw. belegen Mitarbeiter/innen ihre Meinung mit beobachtbaren Beispielen, Bewegtbilder oder Fotografien bilden behauptete Merkmale ab. 
die werbende Figur anhand von Vorgaben agiert. Dass die Erzähler/innen das Bedürfnis haben, die Wahrhaftigkeit ihrer persönlichen Realität zu unterstreichen, zeigt sich in den uRE folgendermaßen: Handelt es sich um ihr Erleben oder ihre Meinung/Bewertung, die auf persönlichem Erleben gründet, setzen die Erzählenden "Trigger" ein, die den Wirklichkeitsbezug untermauern sollen. Sprachlich sind das bspw. Wirklichkeitsmarker (Und das meine ich jetzt nicht nur so, wie man eben sagt, ,ach, den finde ich nett" und „,die ist aber kreativ“, nein, ich meine das wirklich ernst. (Korpusquelle: DTKOM_821.1_WB)). Das verdeutlich zugleich eine grundlegende Unterscheidung: Auf der einen Seite besteht die Unterscheidung zwischen fiktiv und real (Fiktivität und Realität), die sich auf den ontologischen Status der erzählten Inhalte bezieht. Nachweis über den Status gibt der Bezug zur außertextlichen Wirklichkeit. Diesbezüglich ist Werbung fiktiv, während die uRE einen realen Bezug für sich reklamieren. Auf der anderen Seite geht es um die Darstellung des Erzählten. Also darum, ob die Erzählung Anhaltspunkte dafür enthält, dass die dargestellten Sachverhalte imaginär (erfunden) oder wahr sind, unabhängig davon, wie sich der Sachverhalt in der außertextuellen Wirklichkeit tatsächlich verhält. Die Unterscheidung läuft auf die Dichotomie von Fiktionalität und Faktualität hinaus. In den uRE liegt eine faktuale Rede vor, die als „,[a]uthentische Rede“ (Martinez/Scheffel 2007: 188) von einem „realen Sprecher mit behauptender Kraft geäußert [wird]“ (ebd.). Unterstützten sollen das „Trigger“, die sprachlicher und inhaltlicher Art sowie auf die Erzähler/innen bezogen sind. Mit Blick auf die Erzähler/innen ist so ein Trigger bspw. deren Authentifizierung durch eine persönliche Beteiligung an den erzählten Ereignissen (Ich-Erzähler/in) oder Informationsweitergabe aus erster Hand (Zitate), ebenso wie die Mitgliedschaft im Unternehmen mit einer überprüfbaren Rolle (Experte oder Expertin).

In der bisherigen Diskussion habe ich einen wesentlichen Aspekt noch nicht ausreichend berücksichtigt, den Klein mit seinem Konzept des „Geltungsmodus“ (Klein 2000: 36 ff.) erfasst. Klein bezieht sich auf Wunderlich (1976), der bereits feststellte, „daß Sprechakte erwartbare Konsequenzen für die weitere Entwicklung der gemeinsamen Situation der beteiligten Personen haben“ (Wunderlich 1976: 57, nach Klein 2000: 36). Dieser Aspekt ist nicht unerheblich, wenn man bedenkt, dass Unternehmen sich nicht ohne Grund mithilfe der uRE nach außen darstellen. Unter dem Geltungsmodus versteht Klein „Ansprüche, Obligationen und Rechte" (Klein 2000: 36) der Textverfasser/innen und der Adressat/innen, die institutions- und textsortenspezifisch sind. Konkret handelt es sich um die „Bindekraft der Exemplare einer Textsorte“ (ebd. 2000: 37), die im Falle der uRE unterschiedlich zu der der klassischen Werbung ist. Klein schlägt drei Aspekte vor, anhand derer der Geltungsmodus einer Textsorte zu bestimmen wäre: 
- „Selbstbindung der Emittenten und Fremdbindung der Adressaten/ Rezipienten,

- beanspruchte oder prätendierte und tatsächliche Bindekraft,

- Stärkegraden der Bindung.“ (ebd.)

Wendet man Kleins Überlegungen auf die uRE an, folgt daraus: Die Erzähler/innen stellen einen Richtigkeits- und Wahrheitsanspruch (vgl. politische Debatten, Klein 2000: 37) her, „,der einen Ernsthaftigkeitsanspruch impliziert“ (ebd.). Im Falle von Werbung ist das schon aufgrund der Fiktionalisierungstendenz zugunsten der Unterhaltung nicht gegeben. Von der Adressatenseite erwarten Unternehmen in Bezug auf ihre uRE, dass die Adressat/innen das Gesagte ernstnehmen. Während die Werbung dagegen zu starker Übertreibung tendiert, sodass sie gerade nicht beim Wort genommen wird. Tatsächlich kann aber auch bei uRE davon ausgegangen werden, dass von der Adressatenseite aufgrund der ökonomischen Orientierung von Unternehmen eine gewisse Skepsis vorherrscht. Das legt jedoch auch nahe, dass die Unternehmen sich selbst verbindlich an den Geltungsmodus binden, um ihr Geschäft nicht zu gefährden (entspr. der Selbstbindung der Verfasser/in an den Geltungsmodus). Würden sie sich nicht daran halten, würden sie der Täuschung und Lüge bezichtigt, und von der Öffentlichkeit mit einem Image- und Reputationsverlust sanktioniert ${ }^{26}$. Des Weiteren ist die Bindekraft des Geltungsmodus prätendiert/behauptet, da es keine Formalien gibt, die bindend sind (z. B. ein Vertrag). Darauf ist die kritische Distanz der Rezipient/innen zurückzuführen, die den Stärkegrad dieser Bindung aufgrund der wirtschaftlichen Zweckorientierung von Unternehmen anders beurteilen. Das verdeutlicht, dass der vom Unternehmen intendierte Geltungsgrad mit dem von den Rezipient/innen konstruierten Geltungsgrad nicht übereinstimmt, während hinsichtlich der Produktwerbung beide übereinstimmen. Daraus folgt, dass Unternehmen ihren Geltungsanspruch möglichst stark zum Ausdruck bringen. Das machen sie auf der Ebene des Erzähltextes (Wirklichkeitsindikatoren, spezifische Gestaltungsmittel), auf der Ebene der Geschichte (Bezugnahme auf reale Vorkommnisse) und auf

\footnotetext{
${ }^{26}$ Besonders in den sozialen Medien ist diesbezüglich der Shitstorm gefürchtet. Kuhlhüser (2016) ordnet das Phänomen ,Shitstorm“ in die Gattung der Skandale ein. Der Anlass ist das öffentlich werden ,[e]ine[r] Normverletzung, die das kollektive Gefühl der Empörung auslösen kann“ (ebd. 2016: 53) und zu einer kollektiven Empörung im Netz führt. Er entsteht für gewöhnlich spontan und ist geprägt von subjekt-emotionalen Meinungsäußerungen der Kommentatoren. Über einen Shitstorm wird gemeinsam öffentlicher Druck ausgeübt, der sich für Unternehmen als Wirtschafts- und Reputationsschaden auswirken kann. (Kuhlhüser 2016: 53 ff.)
} 
der Ebene der Erzähler/innen (autorisiert durch Unternehmensmitgliedschaft oder Teilhabe an erzählten Inhalten).

Neben den beschriebenen Gemeinsamkeiten von uRE und der klassischen Werbung, die illustrieren, dass in uRE Mittel zum Einsatz kommen, die ,im praktizistischen Verständnis der Werbung zuzuordnen sind" (Krüger 2015: 111), unterscheiden sie sich jedoch auch maßgeblich. Ursächlich dafür ist m. E., dass die Referenzobjekte und die damit einhergehenden Handlungen verschieden sind. In uRE fordern Unternehmen nicht zum Kauf einer Ware/Dienstleitung auf, sondern zum Glauben an die Geschichte bzw. zur Übernahme der vermittelten Botschaft (= Image). Im Hintergrund steht dabei die Existenzsicherung und Legitimation des Unternehmens. Zur Förderung eines positiven Images ist es von Bedeutung, dass die sprachlich geäußerten Propositionen mit Bezugspunkten in der außersprachlichen Wirklichkeit des jeweiligen Unternehmens übereinstimmen oder zumindest damit in eine logische Beziehung gebracht werden. Nur so können die erzählten Inhalte als glaubwürdig erachtet und zugunsten eines positiven Fremdbildes angenommen werden. Hierfür binden sich Unternehmen selbst an einen Geltungsmodus der für sich einen Richtigkeits- und Wahrheitsanspruch auf die außersprachliche Wirklichkeit erhebt. Um das deutlich zu machen implizieren sie auf verschiedenen Ebenen (Erzählakt bei mündl. Erzählungen, Geschichte, Akteure) größtmögliche Authentizität (siehe z. B. Abschn. 9.2.4). Anders als bei fiktionalen Erzählungen, ,die wesentlich durch den imaginären Charakter ihrer Gegenstände gekennzeichnet [sind]" (Genette 1992: 31), gehen wir bei uRE von einem faktualen Geltungsanspruch aus. Für uRE bedeutet das, ein stilles Einvernehmen zwischen Erzähler/innen und Rezipient/innen darüber, dass in den Erzählungen reale Begebenheiten und Fakten aus und über die Unternehmenswelt dargeboten werden, fiktive Elemente gekennzeichnet sind und Sachverhalte in Form von Bewertungen wahr sind, da sie aus der Realität der Erzählenden stammen. Gerade die Unterscheidung des ontologischen Status von Werbung und uRE setzt uRE von der klassischen Werbung ab, was sich wie beschrieben auf verschiedene Weise manifestiert. Auf der anderen Seite bestehen viele Gemeinsamkeiten. Krüger spricht auf Basis einer theoretischen Herleitung im Zusammenhang mit Corporate Storytelling von ,vermeintlich werblichen Formen“ (Krüger 2015: 11). Meines Erachtens lässt sich keine klare Trennlinie ziehen. Aufgrund der Übernahme von werblichen Merkmalen auf der einen Seite und insbesondere den Merkmalen hinsichtlich des Geltungsmodus auf der anderen Seite sind meines Erachtens die Grenzen fließend. Im Grunde liegt eine mehr oder weniger verdeckt werbende Kommunikationsmaßnahme für das nicht wirtschaftsrationale „Unternehmensselbst“ vor. Dabei sind die uRE nicht nur 
nach außen gerichtet, sondern auch ein Identifikationsangebot für die Mitarbeiter/innen, da sie auch deren institutionell geprägten Alltag in den Mittelpunkt rücken. Somit sind auch hier die Grenzen der Kommunikation fließend. Wie ich in Kapitel acht noch zeigen werde, unterliegen die Stilisierungen der jeweiligen Grundtypen einer spezifischen narrativen Rhetorik, die darauf abzielt, die selbstdarstellende Botschaft möglichst überzeugend $\mathrm{zu}$ kommunizieren. Das damit verbundene und pragmatisch orientierte Merkmal der Persuasion, das ebenso auf die Werbung zutrifft, ist für uRE ein grundlegendes Erkennungszeichen. Von der Persuasionsabsicht ablenkende oder verschleiernde Verfahren sind genauso in der Produktwerbung möglich (vgl. Janich 2012: 221). Hinsichtlich der Stilisierung der (S)D wird ebenfalls deutlich, dass die Vereinseitigung, also ,nur die (angeblichen) Vorzüge der beworbenen Sache heraus[zustellen], durchschnittliche Eigenschaften, Mängel oder Unvollkommenheiten aber unerwähnt [zu lassen]“ (Hoffmann 2012: 181), eine weitere Gemeinsamkeit ist. Im Zusammenhang mit den (S)D spreche ich diesbezüglich von der Selbstidealisierung als narrativer rhetorischer Ausprägung. Neben diesen pragmatischen, textuellen und stilistischen Parallelen bestehen nennenswerte Unterschiede aufgrund des Geltungsmodus und dem Entstehungsbereich in den Public Relation. Daher sehe ich die vorliegenden Daten als ein Anzeichen dafür, dass im Rahmen der Unternehmenskommunikation gerade ein neuartiger narrativer Werbehybrid in ,eigener Sache" entsteht.

Open Access Dieses Kapitel wird unter der Creative Commons Namensnennung 4.0 International Lizenz (http://creativecommons.org/licenses/by/4.0/deed.de) veröffentlicht, welche die Nutzung, Vervielfältigung, Bearbeitung, Verbreitung und Wiedergabe in jeglichem Medium und Format erlaubt, sofern Sie den/die ursprünglichen Autor(en) und die Quelle ordnungsgemäß nennen, einen Link zur Creative Commons Lizenz beifügen und angeben, ob Änderungen vorgenommen wurden.

Die in diesem Kapitel enthaltenen Bilder und sonstiges Drittmaterial unterliegen ebenfalls der genannten Creative Commons Lizenz, sofern sich aus der Abbildungslegende nichts anderes ergibt. Sofern das betreffende Material nicht unter der genannten Creative Commons Lizenz steht und die betreffende Handlung nicht nach gesetzlichen Vorschriften erlaubt ist, ist für die oben aufgeführten Weiterverwendungen des Materials die Einwilligung des jeweiligen Rechteinhabers einzuholen.

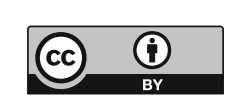

